\title{
Les recherches sur la ville en France : un champ cloisonné ?
}

Retour sur deux exemples d'interdisciplinarité dans la géographie urbaine des années 1970

Urban research in France : a compartmentalized field? A look back at two examples of interdisciplinarity in urban geography during the 1970s

\section{Matthieu Pichon}

\section{OpenEdition Journals}

Édition électronique

URL : https://journals.openedition.org/ress/4022

DOI : $10.4000 /$ ress. 4022

ISSN : 1663-4446

\section{Éditeur}

Librairie Droz

\section{Édition imprimée}

Date de publication : 25 mai 2018

Pagination : 153-179

ISSN : 0048-8046

Référence électronique

Matthieu Pichon, «Les recherches sur la ville en France : un champ cloisonné ? », Revue européenne des sciences sociales [En ligne], 56-1 | 2018, mis en ligne le 25 mai 2021, consulté le 08 janvier 2022 URL : http://journals.openedition.org/ress/4022 ; DOI : https://doi.org/10.4000/ress.4022 


\title{
LES RECHERCHES SUR LA VILLE EN FRANCE: UN CHAMP CLOISONNÉ? RETOUR SUR DEUX EXEMPLES D'INTERDISCIPLINARITÉ DANS LA GÉOGRAPHIE URBAINE DES ANNÉES 1970
}

\author{
MATTHIEU PICHON \\ Université Paris-I Panthéon-Sorbonne \\ matthieu.pichon@parisgeo.cnrs.fr
}

\begin{abstract}
Résumé. II est courant de souligner les différences de structuration des recherches sur la ville entre la France et le monde anglo-saxon: les premières seraient marquées par la segmentation disciplinaire, les secondes par la transdisciplinarité. Sans complètement remettre en cause ce constat, cet article vise néanmoins à le nuancer. Certains travaux réalisés dans les années 1970-1980 se distinguent en effet par une approche transgressant leurs «matrices disciplinaires» d'origine. Les cas de deux géographes de Lyon (Marc Bonneville et André Vant) seront ici évoqués, sur la base d'entretiens et d'une étude des textes, qui permettent d'apporter un éclairage différent sur l'histoire de la recherche urbaine française, en insistant davantage sur l'interdisciplinarité effective (dans l'ordre des référentiels et des pratiques de recherche) que sur son morcellement du point de vue institutionnel.
\end{abstract}

Mots-clés: géographie urbaine, histoire des sciences humaines et sociales, interdisciplinarité, (post-)marxisme, recherche urbaine.

\begin{abstract}
When comparing French and English or American urban research, we often consider that they are opposed in the way they are organized and structured-the former being split into different disciplines, whereas the latter is more transdisciplinary. This article aims at reconsidering this statement by studying some geographers who tried to bypass their original field in different ways, during the 1970s and the 1980s. Based both on interviews and text analysis, this study focuses on two French geographers from Lyon (Marc Bonneville and André Vant), thereby providing a more balanced view of the history of urban research in France: despite the fragmentation between different disciplines dealing with urban issues, there also existed some more interdisciplinary works, whether it be in their theoretical frameworks or in the way they were produced.
\end{abstract}

Keywords: interdisciplinarity, history of the social sciences, (post-)marxism, urban geography, urban studies. 


\section{INTRODUCTION}

Dans un numéro récent des Actes de la recherche en sciences sociales consacré à l'«espace des disciplines », Johan Heilbron et Yves Gingras soulignaient la montée, depuis les années 1990, d’une vague de «débats sur l’interdisciplinarité » visant principalement à «remettre en question les disciplines» considérées comme «des structures insulaires, fermées à la fois vis-à-vis des autres disciplines et des utilisateurs de la connaissance situés hors du monde académique » (Heilbron et Gingras, 2015).

Ces débats touchent aussi des sous-champs plus spécifiques, comme celui des recherches sur la ville. En 20I3, par exemple, la revue Métropolitiques publiait un dossier dont l'éditorial avait pour titre-question «Y a-t-il des "urban studies" à la française?», sur le modèle des «études urbaines» «transdisciplinaires à l'anglo-saxonne» (Collet et Simay, 20I3). Les deux auteurs y soulignaient que «l'histoire des sciences sociales de l'urbain et leur structuration institutionnelle façonnent des productions françaises encore largement disciplinaires ».

Si ces réflexions sur le «cloisonnement disciplinaire» (ibid.) de la recherche française restaient ici analytiques, d'autres discours n'hésitent pas à prendre un tour plus sévère et prescriptif, dénonçant par exemple «les pesanteurs universitaires, les habitudes professionnelles, la paresse intellectuelle ambiante [qui] se combinent pour empêcher toute innovation», et affirmant qu'«il serait temps de rassembler les divers savoirs et savoir-faire sur les villes [...] en un apprentissage transdisciplinaire dont l'intitulé serait "études urbaines" (Paquot, 20I4, p. 99).

Cet article n’a pas vocation à déterminer s'il faudrait ou non développer un champ des études urbaines, ni à juger de l'intérêt ou du désintérêt que cela pourrait représenter. Nous entendons plutôt mettre en discussion les représentations tranchées du champ scientifique français évoquées ci-dessus, et particulièrement les représentations du sous-champ des recherches sur la ville. Certes, le poids des disciplines reste important dans l'organisation du champ scientifique, notamment d'un point de vue institutionnel', mais faut-il voir là le règne définitif et impératif d'un régime disciplinaire strict, ou peut-on apporter des nuances à cette représentation? 
Pour aborder cette question, nous suivons Johan Heilbron et Anaïs Bokobza pour souligner qu'«au lieu de considérer, de façon abstraite, "disciplinarité" et "transdisciplinarité" comme deux modes de production des savoirs opposés, la question de l'ouverture et de la fermeture des domaines de recherche doit être posée de manière relationnelle et relative, et non dichotomique ou typologique, c'est-à-dire comme une question de degré» (Heilbron et Bokobza, 2015, p. I09). Prendre de la distance avec une approche trop dichotomique, qui mettrait face à face deux régimes fondamentalement distincts - le cloisonnement d'un côté, l'ouverture complète de l'autre, permet alors d'observer plus finement des processus d'interdisciplinarisation. C'est là la thèse que nous voulons défendre: même dans un champ scientifique perçu comme compartimenté, même sans l'existence de «studies», il y a bel et bien eu de tels processus dans les recherches françaises sur la ville. En observer la mise en œuvre permet justement de sortir d'une grille de lecture trop binaire.

Il nous faut au préalable définir ce que nous entendons par «discipline». Sans prétendre donner ici une définition complète et définitive, on peut néanmoins considérer que le terme désigne «l'organisation particulière au sein de laquelle se développent les savoirs modernes comme ensemble de pratiques codifiées et reconnues valides par un collectif auto-délimité» (Fabiani, 2006), cet «ensemble» se rapprochant de ce que Thomas Kuhn nomme une «matrice disciplinaire», composée notamment de «généralisations symboliques », d'une «métaphysique », de «valeurs », d'《exercices types » et d'«exemples»(Kuhn, 2008 [1962] $)^{2}$. Une discipline connaît aussi ce qu'il

par le Conseil national des universités (CNU), dont la plupart des «sections», qui décident de la qualification ou non des candidats, sont disciplinaires et définissent des modes de régulation propres à leur discipline - il faut répondre à un certain nombre de critères spécifiques pour être qualifié en sociologie, ou en géographie, ou encore en histoire, etc. Une nuance cependant pour le sujet qui nous concerne: depuis 1992, il existe au sein du CNU une section «Aménagement et Urbanisme», la 24e, qui se revendique comme pluridisciplinaire.

2 Il est difficile ici de revenir entièrement sur tout ce qu'implique la notion de «paradigme». Soulignons simplement que son utilisation fait débat au sein des sciences sociales, certains auteurs considérant - non sans fondement, puisque Kuhn se réfère surtout aux sciences «dures»-que celui-ci s'appliquerait difficilement à ces dernières car elles seraient caractérisées par une «pluralité théorique» (Passeron, 2006 [1991]). Ce qui n'empêche pas nécessairement de s'en inspirer pour rendre intelligible l'évolution d'une discipline de sciences «molles», comme la géographie (Orain, 2009). 
appelle une «tension essentielle» entre stabilisation du savoir, nécessaire à sa reproduction par la transmission, et sa déstabilisation «par remaniement, disqualification et hybridation, et par l'établissement de nouveaux rapports, toujours provisoires, entre les disciplines » (Fabiani, 2006, p. I5). Une discipline se maintient ainsi en reproduisant le savoir sur lequel elle se fonde - notamment par l'enseignement - mais ses logiques internes, épistémologiques et sociologiques, tout comme les «influences externes» (Pollak, 1975) au champ scientifique font peser sur elle un risque de perturbation, qui peut aller jusqu’à sa déstabilisation. Enfin, les disciplines «structurent un ordre institutionnel avec des départements, des procédures de certification, des revues, ainsi que des instances nationales et internationales» (Heilbron et Gingras, 2015, p. 8).

Ainsi, la discipline constitue tout à la fois un ensemble d'institutions, de régulations sociales, de dynamiques sociologiques, et de contenus cognitifs, de méthodologies, de savoirs et savoir-faire. L’interdisciplinarité ${ }^{3}$ désignera alors pour nous un état intermédiaire et dialectique entre inscription et dépassement disciplinaires, au sein duquel les éléments constitutifs de chaque champ disciplinaire rentrent en tension les uns par rapport aux autres.

Cette interdisciplinarité, nous l'observerons depuis la géographie urbaine des années 1970, auprès de deux géographes : André Vant, enseignant et chercheur lyonnais et stéphanois (université Lyon-II puis université de Saint-Étienne), et Marc Bonneville, dont la carrière académique s'est surtout faite à Lyon-II et au sein de l'Institut d'urbanisme de Lyon. Tous les deux formés selon la matrice disciplinaire de la géographie, une discipline alors institutionnalisée depuis plus d’un demi-siècle, mais dans une période où elle connaît ce que Kuhn appellerait une «anomalie». Période de crise et de transformation disciplinaire, les années 1970 constituent pour la géographie française un moment tout à fait opportun pour observer le jeu des disciplines et surtout, des indisciplines.

3 La pluridisciplinarité supposerait plutôt une pluralité de points de vue disciplinaires juxtaposés sur un même objet, et la transdisciplinarité une forte fluidité entre disciplines, voire un effacement important des limites entre ces dernières. II semble que ces notions conviennent moins aux cas étudiés ici. 
Si l'analyse de deux parcours de recherche dans une période circonscrite limite les montées en généralité et ne remplace pas un panorama plus large des dynamiques d'ouverture disciplinaire, elle permet en revanche une mise au jour plus fouillée et détaillée de leur mise en œuvre. Pour autant, notre démarche n’est pas strictement idiographique: en effet, au travers des parcours et des travaux individuels, nous identifions des processus plus généraux qui déterminent en partie les pratiques de recherche. Enfin, ce que nous voulons donner à voir, c'est une forme parmi d'autres d'interdisciplinarisation au sein de la géographie urbaine des années 1970, forme qui pourrait se retrouver chez d'autres, mais qui n'épuise pas à elle seule - loin s'en faut - la diversité des incursions extra-disciplinaires mises en ouvre par les géographes urbains des dernières décennies.

\section{LES ANNÉES 1970:}

\section{UNE PÉRIODE FAVORABLE À L'INTERDISCIPLINARITÉ?}

Les deux parcours de recherches que nous voulons évoquer doivent être réinscrits dans le contexte qui les a vu naître, les années 1970, marquées pour le sujet qui nous intéresse par au moins deux éléments importants : d’une part, des transformations qui ont lieu au sein de la discipline géographique, et, d’autre part, des inflexions dans les politiques de recherche au sein de diverses administrations centrales de l'État et du Centre national de la recherche scientifique (CNRS). Nous faisons l'hypothèse que ces deux éléments ont pu faciliter, voire encourager, sur des questions liées à la ville, une plus grande porosité entre certains géographes et des collègues ou des référentiels théoriques d’autres disciplines.

\section{I.I. OBSERVER L'INTERDISCIPLINARISATION DEPUIS UNE DISCIPLINE EN RENOUVELLEMENT}

Le choix de la discipline géographique présente un intérêt certain pour aborder l'interdisciplinarité à l'œuvre dans les recherches sur la ville. Il nous semble qu'implicitement, le discours sur le «cloisonnement disciplinaire » se focalise souvent avant tout sur la dimension institutionnelle des disciplines. Non sans raison : l'institutionnalisation d'un champ de savoirs joue de fait un grand rôle dans sa codification, sa stabilisation et sa reproduction, tant d'un 
point de vue épistémologique que du point de vue sociologique, et les disciplines des sciences sociales reposent bien sur des institutions différenciées. Néanmoins, cette focalisation sur les institutions peut laisser de côté l'observation des référentiels cognitifs et des pratiques effectives de recherche qui ne coïncident pas toujours avec les découpages institutionnels des «disciplines» constituées - autrement dit, une «matrice disciplinaire» peut connaître des déformations et des écarts sans pour autant que les institutions de la discipline ne s'effacent complètement. Or ceci est sans doute plus prégnant quand il existe une «crise» et une transformation disciplinaire, comme cela a été le cas pour la géographie à la fin des années 1960 et au tournant des années 1970 : si cette dernière, en tant que discipline universitaire, est depuis longtemps ancrée au sein des institutions du champ scientifique et universitaire - plus longtemps, par exemple, que la sociologie - sa «matrice disciplinaire», quant à elle, entame alors un large renouvellement.

Jusquaux années 1970, la géographie universitaire s'organise autour d’un paradigme quasi-unique, qualifié désormais de «classique». La «matrice disciplinaire» de ce paradigme régit tout à la fois les travaux de recherche, l'enseignement, les exercices pédagogiques, le travail de thèse: fondée sur un «réalisme "exhaustiviste" », la géographie classique vise «l'explication causale des relations homme-nature » en même temps que «la compréhension des "personnalités géographiques" » (en cherchant à saisir la singularité de chaque objet - région, pays, milieu, etc.) (Orain, 2006, p. I04)․ La géographie urbaine antérieure aux années 1960-1970, quant à elle, est certes «formatée » par cette matrice mais n'est pas «uniforme» pour autant (Robic, 2003). En effet, «trois principaux schémas rationnels» s'y distinguent: une approche «environnemental[e] ou écologique», «dominant[e]», qui s'intéresse à «l'influence du milieu»; une approche «morphologique ou plus largement paysagique », et une approche «chorologique » où la ville est «étudiée comme l'élément structurant d’un espace régional» (Robic, 2003, p. I27-I28).

4 Pour plus de détails sur ce paradigme «classique» et sa «crise», nous renvoyons au travail d'Olivier Orain (notamment, 2006 et 2009). 
La «crise» que connaît la discipline au début des années 1970 remet en question le paradigme classique et sa matrice et entraîne de «profonds bouleversements » (Orain, 2006, p. 107) théoriques et épistémologiques. Les pratiques de recherche et d'enseignement évoluent : émergence de nouvelles méthodes, développement de formations universitaires rénovées - surtout à partir du milieu des années 1970 - qui ouvrent sur de nouveaux débouchés (Pichon, Leininger-Frézal et Douay, 20I7), lassitude quant aux «recettes traditionnelles » (Orain, 2006, p. I Io) du travail de thèse, etc. D’une façon générale, les années 1970 constituent aussi pour la géographie une période de transition du «génie perso au labo et au réseau» (Robic, 2007): d’une «tradition de recherche associée à une réputation d'excellence individuelle garantie par la thèse d'État», on passe petit-à-petit au «développement d'un travail collectif» et à une «ouverture interdisciplinaire» (ibid., p. I). Ces transformations sont d'ailleurs évoquées par les chercheurs que nous étudions, qui perçoivent la situation préalable à ces renouvellements comme assez rigide :

Ces années-là, c'était la fermeture des disciplines! [...]! Cloisonnées !5

Dans la géographie, le travail en groupe n'était pas une tradition très forte [...]. Avoir une production qui soit une production collective en groupe, en équipe, c'est quelque chose qu'essayait d'instaurer le CNRS, mais la façon dont les gens sont structurés $[\ldots]$ ne facilite pas ça. ${ }^{6}$

L'ensemble des éléments constitutifs de cette «révolution» a finalement conduit à un «foisonnement des abords, des conceptions et doctrines», si bien que «nul ne pourrait $[\ldots]$ prétendre qu'une manière de faire, qu'une thématique, qu’un paradigme, dominent, ne serait-ce qu’en géographie humaine» (Orain, 2003, p. 293).

Il en va de même pour la géographie urbaine, qui s'est engagée elle aussi dans une transformation plurielle, avec l'émergence de méthodes et de référentiels théoriques différenciés renouvelant - au moins en partie - les modalités antérieures de l'étude des villes en géographie. Or cette pluralisation s'est aussi

5 Entretien avec André Vant, 3 et 6 novembre 2015, Saint-Étienne.

6 Entretien avec Marc Bonneville, 4 novembre 2015, Saint-Étienne. 
nourrie et appuyée sur les incursions extra-disciplinaires opérées par un certain nombre de géographes, ce qui peut se comprendre aisément : dans une période de remise en question des pratiques de recherche «classiques», l'inspiration extra-disciplinaire ou internationale (puisant dans d’autres «écoles» nationales de géographie) peut servir de levier à l'aggiornamento de la discipline. Ainsi à partir des années 1970, et pour ne donner que quelques exemples7, faire de la géographie urbaine a pu tout à la fois consister à s'inspirer de la «théorie des structures dissipatives » du physicien et chimiste Ilya Prigogine pour modéliser la ville comme système ouvert et complexe (Pumain, Sanders et Saint-Julien, I989, p. 3-4), qu'à souligner, dans une inspiration lefebvrienne, que l'espace urbain était une «production sociale» issue des rapports de production.

\section{I.2. UNE INFLEXION DES POLITIQUES DE RECHERCHE}

Un autre élément de contexte apparaît important, qui tient dans l'orientation que prend la politique de l'État en matière de recherche au tournant des années 1970. Celle-ci se manifeste notamment dans la préparation du VI ${ }^{\text {e }}$ Plan (DGRST et Commissariat général au Plan. Commission recherche, 197I) et, en prolongement, au sein du CNRS. De manière générale, elle vise à renforcer la programmation de la recherche, y compris en sciences humaines, selon une série d’objectifs et de priorités définis non plus par discipline - comme cela prévalait lors du Ve Plan, qui avait le premier «mentionné [...] l'urgence à développer des recherches portant sur l'urbanisation en France» (Milanovic, 2005, p. I20) - mais au regard de thématiques volontairement plus générales.

Il est ainsi envisagé que les crédits soient répartis selon des groupes sectoriels ou thématiques qui «particip[ent] à une finalité commune ou relèv[ent] d’un même type de préoccupations» (ibid., p. I3). Les «orientations scientifiques » visent explicitement le rapprochement disciplinaire : ainsi le «secteur des sciences de l'homme abandonne la classification en disciplines multiples et

7 La mise au jour des différentes voies théoriques et méthodologiques dans lesquelles s'est engagée la géographie urbaine française depuis les années 1970 demanderait le développement d'une analyse à part entière, qui dépasse le cadre de cet article. C'est néanmoins l'un des objectifs auquel s'attache notre recherche doctorale. 
parcellaires », considérée comme «plus nécessairement pertinente» (ibid, p. 24), pour favoriser des recherches polarisées en axes et en «thèmes interdisciplinaires» (ibid, p. 28-29). Enfin, comme pour le Ve Plan, la question des villes et de l'urbain est largement considérée comme une préoccupation montante: l'urbanisation, l'industrialisation et leurs conséquences sont perçues comme des enjeux forts de la décennie, et imposent de réfléchir à l’amélioration du cadre de vie et des relations entre l'homme et son environnement. Dans ce cadre, le «thème pluri-disciplinaire de la croissance urbaine» (ibid, p. 45) est alors retenu comme l'un des axes de recherche importants du plan quinquennal.

Plus largement, la fin des années 1960 et la première moitié des années 1970 sont marquées par la mise en place et la montée d'une recherche urbaine incitée par l'État (essentiellement par le Commissariat du Plan, le ministère de l'Équipement et la Direction générale de la recherche scientifique et technique [DGRST]) au moyen de contrats de recherche. Si dans un premier temps, ce sont surtout des bureaux d'études qui répondent à cette offre - dans lesquels on retrouve souvent des équipes pluridisciplinaires d’ingénieurs, d’architectes, d'économistes, de géographes et de sociologues - dans un second temps les universitaires vont à leur tour s'engager dans cette voie (Milanovic, 2005).

En résumé, l'orientation générale des politiques scientifiques en ce début des années 1970 comprend à la fois une impulsion vers l'interdisciplinarité, une préoccupation autour des questions d'urbanisation, et une volonté, pour l'État, d'influencer directement le cours de la recherche en programmant les finalités et objectifs généraux de cette dernière, notamment pour la relier aux enjeux contemporains - ce qui, bien sûr, ne préjuge pas de ce qu'en feront effectivement les chercheurs.

L'histoire de la géographie vient alors croiser ici l'histoire des politiques scientifiques, et celle du CNRS en particulier. En accord avec le VIe Plan, celui-ci promeut une politique de recherche contractuelle et entend rattraper le retard accumulé en sciences humaines, notamment en termes d'équipements et de structures d'accueil. Or la transition qui a lieu au sein de la géographie s'appuie sur les dispositifs et instruments développés par le CNRS dans ce cadre. Qu'il s'agisse de l'association des équipes de recherche universitaires (sous la forme de «laboratoires associés » (LA) au CNRS), du recrutement de personnels 
techniques en sciences humaines et sociales, de la mise en réseau de chercheurs (au sein des «recherches coopératives sur programme», les «RCP») ou - invention nouvelle en ce début des années 1970, et directement promue par le $\mathrm{VI}^{\mathrm{e}} \mathrm{Plan}$ - des «ATP», «actions thématiques programmées», tous ces instruments contractuels et financiers concourent à rendre plus collectives les recherches des géographes, et à accroître leurs interactions avec des chercheurs d'autres disciplines (Robic, 2007). Précisons, puisque nous allons en reparler, que les ATP sont directement inspirées des «actions concertées» de la DGRST. Elles visent à orienter les recherches via des appels d'offres et «sont caractérisées par trois principes: une volonté de recherche pluridisciplinaire, le désir d’accroître la contribution des sciences de l'homme aux problèmes contemporains et la mise sur pied d'une procédure souple et rapide» (Collectif, 1999).

L'étude des parcours de recherche d'André Vant et de M. Bonneville illustre le rôle de ce contexte: nous le verrons, les transformations que nous venons d'évoquer se retrouvent dans ces cas particuliers, qui soulignent ainsi par touches successives l'importance de logiques plus structurelles caractéristiques de cet «air du temps».

\section{ANDRÉ VANT ET MARC BONNEVILLE : DEUX CAS D'INTERDISCIPLINARISATION DE LA RECHERCHE SUR LA VILLE

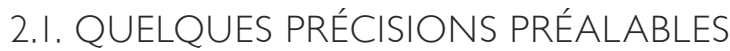

André Vant et Marc Bonneville ont été enseignants et chercheurs à l'université de Lyon-II puis, pour Vant, à l'université de Saint-Étienne à partir des années 1990. Ils appartiennent tous deux à ce que ce dernier a désigné comme une «géographie sociale lyonnaise» (Vant, 1984). Retraçant la généalogie de cette «géographie»-qu'il ne considère pas pleinement comme une «école»- il souligne le rôle important joué par Renée Rochefort ${ }^{8}$. C’est cette

8 Géographe lyonnaise, auteure d'une thèse d'État sur Le Travail en Sicile (1961), elle est souvent considérée comme l'une des figures fondatrices de la «géographie sociale» française. Elle propose notamment en 1963 un «renversement de l'ordre des facteurs» en géographie, qui consisterait à «accorderde l'importance, au groupe humain d'abord, à l'espace ensuite» (Rochefort, 1963, p. 20). 
dernière qui dirige les thèses d'État respectives des deux géographes, qui les réalisent au cours des années 1970: Imagerie et urbanisation pour Vant et Croissance urbaine et changement social pour Bonneville, la première portant sur Saint-Étienne et la seconde sur Villeurbanne. Sans trop entrer dans les détails, et malgré leurs différences, ces deux travaux ont en commun d’appréhender la transformation des espaces urbains à travers une approche sociale ou sociologisante.

Choisir d'étudier ces deux géographes, c'est donc déjà restreindre notre propos à l'une des voies qu'a prise la géographie urbaine dans les années 1970-1980, voie que l'on pourrait résumer très imparfaitement sous le syntagme de «géographie urbaine sociale ». Plus encore, c'est aussi et évidemment réduire notre propos à deux parcours de recherche qui ont leurs singularités, parfois difficilement généralisables à l'ensemble de la communauté géographique. Aussi ce choix est-il redevable de justifications.

La plus importante tient sans doute à ce que Vant, tout comme Bonneville, revendiquent explicitement des postures du dépassement disciplinaire, déclinées sous les figures respectives de la «marge» et de l'«hybridité». De ces positionnements discursifs découle, dans les faits, la mise en œuvre d'une certaine interdisciplinarité qui prend deux formes un peu différentes: l'une relève plutôt d'une démarche individuelle (Vant), tandis que l'autre s'inscrit plus avant dans un travail collectif reposant largement sur les instruments mis en place par les politiques scientifiques évoquées plus haut (Bonneville). Elles présentent néanmoins deux grands points communs: d'une part, elles relèvent toutes les deux d'une certaine tension entre inscription et mise à distance de l'inscription dans la discipline. D’autre part, le dépassement disciplinaire qui opère dans les deux cas doit beaucoup à l'adoption d'un cadre théorique qu'on peut qualifier a minima de marxien, si ce n'est de (post-)marxiste?. Ce cadre, nous essaierons de le voir, impose d’aborder l'espace urbain aussi dans ses dimensions historiques, économiques et sociologiques. Ce faisant, il devient souvent nécessaire d’avoir recours à des outils conceptuels et des méthodes

9 Sous ce terme de «post-marxisme», nous regroupons imparfaitement un ensemble de travaux ou de courants inspirés de Marx ou des marxismes, et souvent distants vis-à-vis d'un marxisme qualifié d'«orthodoxe». 
que l'on va puiser ailleurs, dans d'autres disciplines. Le processus de recherche et la construction théorique et argumentative visible dans le produit final (article, ouvrage) portent alors les traces de ces incursions extra-disciplinaires, qui viennent fissurer l'adhésion de ces chercheurs à la matrice disciplinaire géographique.

\subsection{ANDRÉ VANT: LA MARGE DISCIPLINAIRE COMME NÉCESSITÉ?}

Chez Vant, la tentation interdisciplinaire s'exprime régulièrement sous la figure de la marge, que ce soit dans les textes produits dans les années 1970-1980 ou dans le discours que le chercheur porte sur son travail a posteriori. Ainsi, dès l'introduction de sa thèse d'État (Vant, 198I) élaborée durant les années 1970, il présente son projet de la façon suivante:

En définitive, projet ambitieux, et même trop ambitieux sans doute, car l'incompétence se manifeste souvent lorsqu'il sagit de travailler aux marges de sa propre discipline. [...] Nous náurons pour seule excuse [...] que de nous être aventuré en des champs nouveaux "pour le développement futur de la géographie $e^{10 ”}$ (ibid., p. 12).

Cette «aventure », si elle peut relever d'une curiosité personnelle du chercheur, tient aussi d'une nécessité face à une matrice disciplinaire considérée comme insatisfaisante. La genèse intellectuelle de cette thèse donne à voir deux insatisfactions principales. La première, commune à de nombreux géographes de cette génération, se traduit par la mise à l'écart du paradigme «classique» et de la méthode d’analyse des villes qui prévaut avant les années $1970^{11}$. Si certains géographes trouvent dans la géographie «théorique et quantitative» un moyen de dépasser la matrice classique et de refonder la discipline, ces méthodes nouvelles n'emportent pas l'adhésion de Vant ${ }^{12}$. À l'en croire, c'est

10 La citation est de Wolfgang Hartke, géographe allemand (1908-1997).

IIVant rappelle ainsi sa volonté de mettre à l'écart «le déterminisme de Raoul Blanchard», «le possibilisme de Vidal de la Blache», ainsi que «l'organicisme» (Entretien avec André Vant, 3 et 6 novembre 2015, Saint-Étienne).

12 À propos du «quantitativisme pur et dur», il souligne ainsi: «j'en suis revenu totalement à une autre lecture. Ces méthodes je les avais lues dans les Annales de l'Association of American Geographers: je me suis dit "il faut que tu te trouves autre chose" » (Entretien avec André Vant, 3 et 6 novembre 2015, Saint-Étienne). 
«dans la déviance $[\ldots]$ dans la marginalité que l'on innove, toujours », «c'est toujours aux marges que l'on progresse $»^{13}$. Or la marginalité ici est double: vis-à-vis de la matrice disciplinaire traditionnelle, et vis-à-vis de l'une des matrices émergentes de la «nouvelle géographie». Ici prend sens l'idée d'une nécessité interdisciplinaire : face à une géographie classique et une géographie quantitative qui ne donnent pas satisfaction, il devient nécessaire d’aller trouver des schèmes d’intelligibilité et des concepts, référentiels et méthodes qui viennent d'ailleurs, notamment de l'économie, de la sociologie et de l'histoire.

Ce décentrement intellectuel, néanmoins, n’est pas exempt d'une certaine ambivalence, qui apparaît dans le «projet» de cette thèse. Celui-ci semble pris en tension entre deux forces opposées : l'une, centrifuge, encourage le dépassement des limites disciplinaires tandis que l'autre, centripète, ramène à la discipline d'origine. Plus encore, ce pas de côté se projette dans une perspective de renouvellement, de «développement futur» de la discipline. Ici, la «marge» est cette position qui recompose le rapport des disciplines entre elles: sans les faire disparaître, elle crée toutefois une tension ambivalente entre inscription dans la discipline d'origine et dépassement de celle-ci.

\subsection{Une recherche individuelle nourrie par des socialisations informelles}

La production intellectuelle de Vant est visiblement marquée et nourrie (dans sa construction, ses références implicites ou explicites, mais aussi dans le paratexte qui l'accompagne - notes de bas de page, bibliographie) par une lecture approfondie d'une littérature scientifique qui ne relève pas de la géographie telle qu'elle se déployait à l'époque. Cette familiarité avec des écrits extra-disciplinaires témoigne d'un parcours de socialisation particulier, fondée principalement sur une fréquentation plutôt «informelle d de différentes équipes de sociologie, d'économie et d'histoire lyonnaises ou stéphanoises : 
Quelque chose qui a été important dans mes rapports, ça a été aussi le CREUSET $^{4}$, à Saint-Étienne, qui était le centre de recherche des économistes. [...] C'était [...] des personnes qui réfléchissaient, qui quelques fois me donnaient des idées [...] des économistes qui eux aussi voulaient s'ouvrir sur les sciences sociales. ${ }^{15}$

Je travaillais avec des gens avec lesquels je pouvais discuter et qui mapportaient toujours une vision nouvelle ou une recherche nouvelle, que je ne connaissais pas, parce que moi les revues d'économie, si je n’avais pas eu ces amis-là, je n'y serais pas allé, les revues de sociologie de la même manière. Et je me servais de leurs bibliothèques. Et au $\mathrm{CRESAL}^{16}[\ldots]$ je voyais ce qu'ils lisaient eux, alors je me suis mis à lire Lojkine, Préteceille, Topalov [...]. Je lisais toute cette littérature, et je suis resté là-dedans jusqu'à ma soutenance de thèse. ${ }^{17}$

Ces interconnaissances - facilitées dans les années 1970 par la taille encore modeste du champ universitaire local - sont décrites comme des liens amicaux, bien plus que comme des dispositifs institutionnalisés et stabilisés de coopération scientifique - exception faite cependant de l'ATP pluridisciplinaire «Observation du changement social» lancée par le CNRS en 1975 et à laquelle il participe $^{18}$. Peu de recherches collectives, donc, mais tout un jeu de sociabilités plus informelles, qui n'ont pas moins d'importance dans le parcours intellectuel de Vant. Non seulement ces discussions informelles permettent d'échanger, de faire émerger de nouvelles «idées » de recherche, mais elles permettent encore l'accès à tout un champ de productions scientifiques et théoriques auxquelles Vant va s'acculturer - et notamment la sociologie urbaine marxiste. À ce titre, la fréquentation du CRESAL revêt peut-être un caractère particulier puisque

I4 II s'agit du Centre de recherches économiques de l'université de Saint-Étienne, au sein duquel travaillaient notamment plusieurs économistes que l'on retrouve dans le projet financé par l'ATP «Croissance urbaine» et évoqué plus bas.

15 Entretien avec André Vant, 3 et 6 novembre 20I5, Saint-Étienne.

16 Centre de recherches et d'études appliquées de la Loire, qui a produit de nombreuses études sociologiques sur Saint-Étienne, abondamment citées par Vant dans ses propres travaux. II semble qu'il ait collaboré à l'une d'entre elles en 1973, Étude exploratoire sur les processus d'évolution des grands ensembles.

17 Entretien avec André Vant, 3 et 6 novembre 2015, Saint-Étienne.

I8 II publie notamment un article intitulé «Valbenoîte, du quartier éclaté au quartier investi» dans le volume XVI des Cahiers de l'Observation du changement social, en 1982. 
celui-ci entretient des liens déjà notables avec la recherche urbaine nationale ${ }^{19}$, où se déploient nombre de travaux de sociologues marxistes.

Un autre point important tient dans le parcours universitaire de Vant. Si la dimension sociologisante du travail de ce dernier rappelle la volonté de mettre le «social» avant le «spatial» en géographie, comme le revendique Rochefort (1963) qui dirige sa thèse d’État (en lui laissant néanmoins une grande liberté), ce n'est sans doute pas l'influence la plus déterminante dans la formation intellectuelle du chercheur. Il faut chercher surtout du côté d'Osiris Cecconi, sociologue, professeur de philosophie en classe préparatoire au lycée Fauriel de Saint-Étienne au moment où Vant y est étudiant, et préfacier de la version publiée de sa thèse. Vant dit avoir été «très marqué » par celui qu'il qualifie de «communiste» ou de «marxiste orthodoxe». Il souligne notamment: «C'est vrai que je considérais que c'était mon maître: ce n’était pas un géographe qui avait été mon maître $[\ldots]$ c'était lui, en philo. $\gg^{20}$

Cette influence vient se surajouter au contexte idéologique et universitaire de l'après-1968. Non seulement « 1968 》 a eu des répercussions sur la géographie (Orain, 20I5), mais il a aussi plus largement contribué à l'émergence d'un paysage intellectuel critique. «On était très marqués à cette époque en tant qu'étudiants par toute l'idéologie marxiste $»^{21}$, indique Vant, mais aussi par d'autres travaux comme ceux du CERFI ${ }^{22}$, qu'il a aussi largement consultés.

Le parcours scolaire, la rencontre avec des «maîtres», la fréquentation de collègues non-géographes jouent ainsi un rôle important dans l'émergence d'un travail où l'interdisciplinarité est prégnante : en bref, mais c'est un point fondamental dans une perspective d'histoire des sciences, ces éléments

19 Le Centre a obtenu au moins 10 contrats de recherche au cours des années 1970, notamment auprès du ministère de l'Équipement (ce chiffrage s'appuie sur les Fiches analytiques de la recherche urbaine publiées par le Centre de documentation sur l'urbanisme à partir des années 1970, qui a fait un travail de recensement des contrats de recherche urbaine passés par différentes administrations de l'État).

20 Entretien avec André Vant, 3 et 6 novembre 20I5, Saint-Étienne.

21 Idem.

22 Centre d'études, de recherches et de formation institutionnelles, collectif de recherche fondé en 1967 par Félix Guattari. 
permettent de rappeler que, y compris dans la recherche, ce qui semble relever de la stricte individualité intellectuelle, voire du «génie perso», doit souvent beaucoup aux socialisations multiples que connaissent les individus.

2.2.2. Le (post-)marxisme comme moteur et opérateur d'une interdisciplinarité cognitive?

Une fois mise en exergue l'importance de ces socialisations, il nous reste à tenter de caractériser brièvement de quoi est constituée ce que nous considérons comme l'approche interdisciplinaire de Vant. Comme on peut le pressentir à la lecture des éléments évoqués plus haut, cette approche s'inscrit pour une bonne partie dans un cadre théorique (post-)marxiste. Ce cadrage est perceptible de façon régulière dans les travaux de Vant, a minima dans ceux des années 1970 et 1980. S’il ne nous est pas possible de revenir sur toute sa production intellectuelle, nous pouvons nous concentrer sur un exemple: l'un des premiers articles à porter la trace explicite de cette approche (post-) marxiste date de 1973 (Vant, 1973). Il s’y intéresse à la conflictualité sociale dont le centre-ville de Saint-Étienne fait l'objet, opposant notamment un «centre prolétarisé » (ibid., p. I I) à un «centre bourgeois» (ibid., p. I5). L'introduction comporte, du point de vue de notre réflexion sur l'interdisciplinarité, un passage très intéressant :

n'est-ce pas au géographe de comprendre la lutte dont l'espace est l'enjeu entre les différents groupes sociaux? Si l'on souscrit à l'hypothèse de travail que les nuances spatiales de localisations et de fréquentations dérivent des structures sociales ${ }^{23}$, ce propos nécessite deux analyses préalables [...]. Il s'agit en fait de reconnaître au travers des disparités spatiales, deux temps distincts: un temps synchronique appartenant à la dynamique interne de chaque groupe social et un temps diachronique exprimant la résultante des confrontations entre différents groupes ${ }^{24}$ (ibid., p. 6).

23 Vant fait référence en note à un article de Manuel Castells, en 1969, «Le Centre urbain. Projet de recherche sociologique» (in Cahiers Internationaux de Sociologie, 46, p. 83-106).

24 Cette fois-ci, c'est Lire le Capital de Louis Althusser et Étienne Balibar, qui est cité en note (tome II, Maspéro, 1968, p. 205-217). 
On y retrouve la tension évoquée au départ entre une inscription disciplinaire en géographie et l'introduction d'un cadre théorique qui n’en relève pas. En effet, la dynamique d'ensemble de l'article découle d'une «hypothèse de travail» qui vient directement des réflexions de Manuel Castells. Cette compréhension de «la lutte dont l'espace est l'enjeu» par le «géographe» se fait paradoxalement à partir d’un schème d'intelligibilité qui relève de la sociologie urbaine marxiste. Plus encore, la référence à l'ouvrage de Louis Althusser et Étienne Balibar qui consiste, grosso modo, en une relecture du Capital de Marx, sert à asseoir et justifier l'analyse du centre par les conflits sociaux dont il fait l'objet. Le centre, comme espace, devient ainsi en partie «la résultante des confrontations» dans un «temps diachronique »: on est là pleinement dans une approche dialectique et historicisée. Afin de montrer que «le centre urbain, qui désigne à la fois un espace géographique et un contenu social, n'existe pas en soi mais en tant que résultat d'un processus social d'organisation de l'espace urbain» (p. 19), il est amené à en passer par une histoire dialectique de la formation du centre-ville, pétrie d'oppositions de classe, de contradictions, qui finissent par produire le centre-ville.

\subsubsection{Vers une approche holiste de l'espace urbain}

Il nous faut aussi évoquer - trop succinctement et partiellement - l'approche que Vant développe dans sa thèse. Consacrée à la question de l'imagerie (la production d'images) de la ville de Saint-Étienne et son rôle dans la production de l'espace stéphanois, elle met là aussi l'accent sur le caractère conflictuel de ces images et contre-images. Le cadre général qui sert de socle à son approche ravive le paradoxe entre discipline et indiscipline. Revendiquant une «perspective de géographie dite sociale» (Vant, 198I, p. Io), il définit celle-ci comme permettant «de poser les problèmes dans un contexte de totalité sociale, c'est-à-dire par rapport à l'ensemble des rapports sociaux, infra et superstructurels, envisagés d'un point de vue statique et d'un point de vue dynamique ». Citant Fernand Braudel, il considère alors que ce cadre saisit «l'homme vivant, complexe, déroutant [...]. L’homme, que la géographie comme toutes les sciences du social, doit se garder de découper en tranches, 
si habile ou artistique soit le découpage $»^{25}$ (ibid., p. I I). Ces éléments font écho au discours qu'il tient a posteriori sur sa démarche: «Et puis toujours revenir à l'espace, mais pour moi la dimension spatiale de la géographie n'est peut-être pas suffisante : je veux dire que ce n'est qu'une dimension. Pour moi, c'est bien le social, le social global, holiste, qui est important. $\gg{ }^{26}$

La géographie sociale, telle qu'il la présente, est une géographie qui ne se limite pas à sa propre matrice - dont l'un des fondements serait sans doute «l'espace ». Elle doit opérer un nécessaire dépassement car l'espace n’est qu’une dimension du social, et que ce dernier est un tout et oblige à une démarche holiste. Il faut donc prendre «l'homme» (mais l'on pourrait aussi dire la société, ou bien encore l'espace urbain) comme un tout à plusieurs dimensions, dont aucune ne mérite d'être laissée de côté. D’où des recours à l'histoire, à l'économie, à la sociologie; d'où des pratiques qui sont parfois plus proches de celles d'autres disciplines (à titre d'exemple, la thèse de Vant repose beaucoup sur un important travail historien de dépouillement et d’analyse d’archives). Ainsi, cette géographie sociale, par sa définition même, autorise voire même impose son propre dépassement disciplinaire.

\subsection{MARC BONNEVILLE, UNE INTERDISCIPLINARITÉ COLLECTIVE}

Marc Bonneville pratique une interdisciplinarité sensiblement différente, notamment parce qu'elle ne se traduit pas seulement dans l'ordre cognitif, mais aussi en termes de pratiques collectives de recherche. Sa thèse, soutenue en I98I, porte dans son titre même la marque d'un investissement dans des recherches collectives et interdisciplinaires sur programme : traitant du «changement social» et de la «croissance urbaine» à Villeurbanne (Bonneville, 198I), elle rappelle ainsi les deux ATP auxquelles Bonneville a participé dans les années I970 - l’ATP «Croissance urbaine» et l'ATP «Observation du changement social et culturel»:

25 Citation qu'il reprend en 1984 dans son article de présentation de la «géographie sociale lyonnaise», et qu'il met en opposition avec le «plaidoyer de Maurice Le Lannou [(1906-1992) géographe professeur à Lyon puis au Collège de France] en faveur d'une géographie autonome, à objet propre et vocation distincte». Autrement dit, la citation resservira dans cet article à appuyer la défense d'une géographie qui soit pleinement une science sociale à vocation totalisante.

26 Entretien avec André Vant, 3 et 6 novembre 20I5, Saint-Étienne. 
J'ai travaillé surtout sur des programmes, des recherches qui étaient des projets de recherche $[\ldots]$, de la recherche urbaine avec d'une part et d'abord, des économistes - c'est très important [...] parce que c'est une façon d'ouvrir le champ de la discipline. [...] Du point de vue théorique et épistémologique, c'était fondamental de se positionner par rapport à l'économie, et l'économie foncière entre autres, l'économie de la ville. [...] Puis dans une autre ATP, j’ai travaillé avec les sociologues, et donc ça donnait une ouverture intéressante avec deux disciplines connexes avec lesquelles on avait affaire. ${ }^{27}$

De fait, bien plus que Vant, Bonneville entretient des relations interdisciplinaires formalisées, puisqu'elles relèvent de contrats signés entre des financeurs (généralement, le CNRS) et des universités ou laboratoires dans le cadre des politiques de recherche que nous évoquions plus haut. Il s'agit ainsi, dans une certaine mesure, du croisement entre une interdisciplinarité horizontale - la volonté de M. Bonneville, comme bien d'autres, de s'ouvrir à d'autres disciplines - et d'une interdisciplinarité verticale et descendante, c'est-à-dire promue, mise en ouvre, cadrée par l'État ou les divers organismes qui relèvent de lui. Ces différentes ATP, et la production intellectuelle qui en découle, ont donc beaucoup à voir avec un gouvernement de la recherche dont l'instrument principal est financier et contractuel: ces recherches, sélectionnées sur la base d'une proposition dans le cadre d'un appel d'offres, obtiennent un budget spécifique pour financer équipements, personnels contractuels et fonctionnement.

\subsection{Au sein de I'ATP Croissance urbaine: un projet d'économistes}

Nous avons choisi de nous arrêter particulièrement sur l'ATP «Croissance urbaine », qui donne lieu à la publication par Bonneville de Villeurbanne : naissance et métamorphose d'une banlieue ouvrière processus et formes d'urbanisation (Bonneville, I978). L'ouvrage est la version publiée du rapport qu'il a rendu dans le cadre de l'ATP. L’introduction en présente les objectifs :

Ce travail doit être vu comme une étude de géographie urbaine. Il s’efforce de saisir simultanément les deux composantes qui font la ville, à savoir la société locale dans ses expressions et ses déterminations multiples, et la matérialité de la ville, c'est-à-dire ses caractéristiques architecturales, physiques, de densité, 
de localisation, etc. Ces deux aspects sont abordés ensemble comme le résultat d'une «production» sociale, qui produit ou reproduit à la fois, mais selon des modalités particulières, la société et le cadre urbain (ibid., p. 7).

En passant trop vite sur ce passage et en considérant que l'auteur s'inscrit simplement dans le cadre disciplinaire de la «géographie urbaine», on manquerait un élément fondamental, que seul un retour sur la genèse du projet proposé pour l'ATP permet de mettre en exergue. Cette recherche est élaborée, proposée et conduite par des économistes de Saint-Étienne (ceux du CREUSET évoqué par Vant) et de Lyon, sous la direction de l'économiste Pierre Dockès. Bonneville, identifié comme un géographe intéressé par la ville et ouvert à une approche économique, est sollicité par cette équipe d'économistes. Le projet déposé répond à l'une des sous-thématiques délimitées par le CNRS: «analyse et perspective de la consommation de l'espace selon les formes d'urbanisation ». Toute la recherche va consister en un renversement de problématique et ce dès le dépôt du projet: plutôt que de partir de la «consommation de l'espace», l'équipe préfère une approche par la «production de l'espace». Le cadrage théorique de la recherche s'organise autour de cette dernière idée.

\subsubsection{La notion de "production de l'espace» comme opérateur d'interdisciplinarité}

Le syntagme de «production de l'espace» fait se croiser deux références théoriques : à la fois l'économie hétérodoxe de John K. Galbraith - critique de la théorie néo-classique du consommateur - et dans le même temps le travail d’Henri Lefebvre, qui publie La Production de l'espace en $1974^{28}$. Ce renversement de problématique est affirmé dès le rendu du projet de recherche ${ }^{29}$ : celui-ci comprend d'abord une critique de travaux existants, où l'on trouve plusieurs dénonciations du «néo-classicisme» en économie. Puis vient la présentation d’une «problématique» qui s'ouvre sur la question de l'influence du «mode de production capitaliste»sur le modelage de l’espace. Références multiples

28 Ces informations sont tirées d'un entretien avec Pierre Dockès, le 20 novembre 20I5, par téléphone.

29 Consulté aux archives du CNRS, Gif-sur-Yvette, fonds n I de l'ATP «Croissance urbaine». 
aux travaux de Castells, Lefebvre et, plus directement encore, de Marx donnent une tonalité largement marxisante à ce projet qui place au centre de son appareil théorique l'idée que l'espace urbain est produit socialement, non seulement comme lieu d'expression des rapports sociaux (du capitalisme) mais aussi comme espace matériel. Dockès reconnaît d'ailleurs à ce propos que, s'il était pour sa part «clairement influencé par les idées de Marx» au moment de la rédaction du projet, «tout le monde» était de toute façon «marqué par l'ambiance $»^{30}$ sinon marxiste du moins marxienne de l'époque.

Si nous pourrions développer plus encore l'«approche théorique p proposée par ce projet d'ATP, ces quelques éléments suffisent déjà pour prendre conscience de ce que la réflexion de Bonneville doit à des pratiques de recherches collectives et interdisciplinaires. Car l'un des points fondamentaux ici tient au fait que ce dernier n’a pas participé à l'élaboration du projet ni de l'approche théorique proposée, puisqu'il a été associé au projet ex-post. Le cadre théorique de son «étude de géographie urbaine » relève donc d'une élaboration qui vient d'économistes. Ce fait-là, Bonneville le revendique d'ailleurs dans l'avant-propos de son ouvrage :

L'étude que l'on présente ici [...] constitue en fait, pour une large partie, le contenu d'un rapport de recherche réalisé $[\ldots .$.$] dans le cadre d'une ATP [...].$ L’essentiel de la problématique est dû à la réflexion collective de l'équipe de recherche dirigée par Pierre Dockès [...]. Si nous revendiquons la responsabilité de l'exposé des faits, de leur organisation et des conclusions, c'est bien la réflexion collective qui donne sa cohérence à cette étude et qui en constitue l'apport le plus original. (Bonneville, 1978, s.p.).

Ainsi, et dans la mesure où «il pouvait permettre de conjuguer les approches économiques, sociologiques et géographiques de l'espace $»^{31}$, le cadre théorique de la «production de l'espace » a constitué un opérateur d'interdisciplinarité pour cette recherche.

30 Entretien avec Pierre Dockès, le 20 novembre 20 I5, par téléphone.

31 Entretien avec Marc Bonneville, décembre 2015, par courriel. 


\subsubsection{Frontières, hybridation et remise en cause de l'autonomie de la géographie}

Si l'on revient à la première citation de Bonneville, l'«étude de géographie urbaine» dont il est question apparaît, comme chez Vant, prise dans une tension entre inscription disciplinaire et cadre théorique et pratiques de recherche qui dépassent les limites de la géographie du moment. Comme il l'indique en entretien :

J'ai toujours pensé que c'était plus productif de travailler en groupe d'une part, deuxièmement de travailler sur les limites, les frontières, on a toujours à y gagner $[\ldots$. Ces frontières ne sont pas des frontières, elles ne sont des frontières que parce que justement, elles permettent de les franchir et de faire le lien, de faire le passage. [...] Est-ce qu'on est capable de travailler sur un champ comme ça [la ville] en nayant pas des concepts de sa discipline? Moi la réponse elle est évidemment oui, parce qu'il faut remettre ces concepts de la discipline en jeu, il faut les affronter, les confronter. Ce n'est pas une question de pureté idéologiquequi consisterait à dire "est-ce que je suis dans les clous de ma discipline ?"32

D’une manière générale, il semble que Bonneville aille plus loin que Vant dans la remise en cause de son inscription disciplinaire. Cela passe notamment par la revendication d'un statut d' «hybride», qu'il affirme à plusieurs reprises : «J'ai toujours été dans cette hybridation [...], du point de vue épistémologique cela me paraît être absolument nécessaire. [...] J’ai toujours un peu réfléchi comme cela : j’ai un objet, j’ai une question, comment je l'aborde? Alors après je vais mobiliser des champs qui sont vastes: je suis vraiment un hybride, vraiment $»^{33}$.

Mais cette hybridation pousse plus loin encore: son attachement au cadre théorique de la «production de l'espace» va l'amener à mettre en cause, plus fortement, l’autonomie disciplinaire de la géographie.

À l'occasion du Géopoint de 1978 - l'un des colloques réguliers organisés par le Groupe Dupont ${ }^{34}$ - Bonneville co-anime avec Vant un atelier sur «le concept de production de l'espace» (voir Géopoint, 1978, p. I79 et suiv.). Bonneville présente un rapport introductif, dont le contenu résonne fortement avec le cadre

32 Entretien avec Marc Bonneville, 4 novembre 20I5, Saint-Étienne.

33 Idem.

34 Collectif de géographes fondé dans les années 1970. De façon générale, les Géopoint constituent 
théorique proposé au sein de l’ATP «Croissance urbaine». Inscrite dans le cadre particulier d'un forum de discussion épistémologique et théorique, dans un contexte de renouvellement de la géographie, son intervention est constellée de réflexions qui, par le biais du «concept de production de l'espace», en viennent finalement à questionner la discipline elle-même. Se demandant si «le primat reconnu à l'économie politique ou simplement à la détermination sociale dans la production des espaces laisse [...] encore place à une science et à des théories proprement géographiques?» (ibid., p. I84), il est «amené à douter qu'un tel projet soit possible». Il propose malgré tout de «juge[r] sur pièces» afin de voir «comment le concept de production de l'espace est [...] utilisé ou plutôt pourrait [...]l'être» (ibid, p. I85) en géographie. Finalement, «à travers l'analyse du concept de production de l'espace et de son usage en géographie», c'est «la question du statut de la géographie dans les sciences sociales » (ibid., p. 187) qui est abordée, et qui en implique une autre:

La géographie doit-elle abandonner l'ambition de se constituer en science autonome? Si son objet est clairement défini, ses concepts et ses références théoriques ne relèvent-ils pas fondamentalement des problématiques des sciences sociales? Reconnaître l'importance de sa contribution, de sa vision, de son objet nous autorise-t-il pour autant à la vouloir scientifique à n'importe quel prix et même à son détriment? (ibid., p. I88).

La réflexion sur le «concept de production de l'espace» opère ainsi non seulement comme une interrogation per se mais aussi plus largement comme un questionnement sur l'autonomie disciplinaire de la géographie. Ce «concept » permet de rapprocher - dans l'ordre des cadres théoriques - la géographie des autres «sciences sociales ». D'une certaine manière, c'est finalement seulement sur des objets différenciés que se fonderait la division des sciences sociales en disciplines autonomes, et non pas sur les concepts ou théories qui, quant à eux, procéderaient de problématiques communes. La discipline «géographie» aurait ainsi son objet - on peut supposer qu'il s'agit de l' «espace» - mais dont 
la compréhension et l'analyse ne sauraient relever d'une théorie proprement géographique, c'est-à-dire interne à la discipline. L'espace de la géographie, c'est «l'espace naturel approprié par la société [...] résultats de procès de production divers mais socialement et historiquement déterminés ». Espace socialisé, espace produit «selon l'acception économique du terme» (ibid., p. I82).) mais aussi espaces «produits sociaux, culturels, symboliques» (ibid., p. 183), l'espace relèverait ainsi bien des «sciences sociales».

\section{CONCLUSION}

Les deux géographes que nous avons évoqués doivent évidemment être considérés comme des exemples parmi d'autres des processus d'interdisciplinarisation qu’a connus la géographie française. Certainement trop partiels pour être représentatifs, ces cas autorisent néanmoins à formuler quelques analyses ou hypothèses.

Ces deux chercheurs ont en commun d'avoir mis en ouvre un certain dépassement de la discipline qui les avait formés. Dépassement facilité par plusieurs éléments de contexte: une période que l’on pourrait qualifier après Kuhn de «révolutionnaire» pour la géographie française, une popularité de théories inspirées du marxisme et qui ouvrent sur l'économie, l'histoire et la sociologie, des politiques de recherche qui encouragent l'ouverture des disciplines. Cet ensemble de facteurs permet de considérer que les élans interdisciplinaires des années 1970 ne relèvent pas nécessairement du hasard, ni de la simple curiosité intellectuelle de tel ou tel chercheur isolé.

Ces exemples permettent de nuancer quelque peu les affirmations qui singularisent la recherche française sur la ville comme compartimentée et fragmentée entre disciplines. Bien sûr, il ne s’agit pas de réfuter radicalement cette prégnance des disciplines «urbaines » ni les résistances ou replis qu’elles ont pu opposer à l'interdisciplinarité. Et, y compris ici, nous avons pu souligner que le dépassement disciplinaire n’est pas toujours tranché, alternant ainsi avec une rémanence de l'inscription géographique de départ. 
Il s'est agi cependant pour nous d'aller contre une forme d'essentialisation des disciplines. En rétablissant ces dernières dans leur densité historique, en redonnant à voir les dynamiques internes, les mouvements, les processus parfois contradictoires qui les animent, on peut porter un regard moins définitif sur la recherche française sur la ville, et sortir d'une opposition dichotomique entre «cloisonnement» et «ouverture». En l'occurrence, il y a bel et bien eu des formes d'hybridation, d'interdisciplinarisation des recherches, à la fois d'un point de vue strictement cognitif - les cadres théoriques - que du point de vue des pratiques de recherches - travail collectif, échanges disciplinaires, etc. Et ce y compris dans une discipline comme la géographie qui dans les années 1970, d’un point de vue institutionnel, est ancrée dans le champ universitaire et dispose de ses propres instances de régulation depuis plusieurs décennies.

Ainsi, au travers des deux cas analysés dans cet article, nous avons voulu mettre en avant deux précautions fondamentales quant à l'étude de la structuration de la recherche urbaine française. En premier lieu, la structuration institutionnelle des disciplines ná pas empêché la mise en œuvre d’une interdisciplinarité en pratique qui demande, pour être observée, que l'on n'en reste pas à la seule analyse des formes de l'organisation du champ scientifique. Ensuite, et surtout, l'alternative entre «cloisonnement disciplinaire» et inter- ou trans-disciplinarité n’est sans doute pas le meilleur schème pour rendre compte de l'histoire de la recherche urbaine : le parcours des deux géographes étudiés ci-dessus, entre ouverture et inscription disciplinaire, montre ainsi que ces questions peuvent être approchées dans une perspective plus dialectique, mieux à même d'en mettre au jour toutes les tensions.

\section{BIBLIOGRAPHIE}

BONNEVILLE M., 1978, Villeurbanne: naissance et métamorphose d'une banlieue ouvrière processus et formes d'urbanisation, Lyon, Presses universitaires de Lyon.

-, 198I, Croissance urbaine et changement social: le cas de Villeurbanne dans l'agglomération lyonnaise, Thèse d'État sous la direction de Renée Rochefort, Université Lumière Lyon 2. 
COLLECTIF, 1999, «Le CNRS et Hubert Curien de 1969 à 1973», La Revue pour I'histoire du CNRS, I, en ligne (consulté le 10 janvier 2018), [en ligne]: $<$ http://journals.openedition.org/histoire-cnrs/488>.

COLLET A. et SIMAY P., 20I3, «Y a-t-il des "urban studies" à la française?», Métropolitiques, [en ligne, consulté le 10 janvier 2018]: <http://www.metropolitiques.eu/Y-a-t-il-des-urban-studies-a-la.html>.

DGRST, Commissariat général au Plan. Commission recherche, 197I, Rapport de la Commission Recherche: préparation au V|e plan, Paris, La Documentation française.

GÉOPOINT, 1978, Concepts et construits dans la géographie contemporaine: Géopoint 78, Lyon, 18-19 mai 1978, Groupe Dupont (dir.), Avignon, Centre littéraire universitaire.

FABIANI J.-L., 2006, «À quoi sert la notion de discipline?», in J. Boutier, J.-Cl. Passeron et J Revel (dir.), Qu'est-ce qu'une discipline?, Paris, Éditions de l'EHESS, p. II-34.

HEILBRON J. et BOKOBZA A., 2015, «Transgresser les frontières en sciences humaines et sociales en France», Actes de la recherche en sciences sociales, 210-5, p. 108-121.

HEILBRON J. et GINGRAS Y., 20I5, «La résilience des disciplines», Actes de la recherche en sciences sociales, 210-5, p.4-9.

KUHN Th. S., 2008 (1962), La Structure des révolutions scientifiques, Paris, Flammarion.

MILANOVIC F., 2005, «Travail organisationnel et institutionnalisation des sciences sociales. Le cas de la recherche urbaine française», Revue d'histoire des sciences humaines, 12-1, p. I17-139.

ORAIN O., 2003, Le Plain-pied du monde: postures épistémologiques et pratiques d'écriture dans la géographie française au vingtième siècle, Thèse de doctorat sous la direction de Marie-Claire Robic, Université Paris I Panthéon-Sorbonne.

-, 2006, «La géographie comme science. Quand "faire école" cède le pas au pluralisme», in M.-C. Robic (dir.), Couvrir le monde: un grand xxe siècle de géographie française, Paris, Association pour la diffusion de la pensée française, p. 90-122.

-, 2009, De plain-pied dans le monde: écriture et réalisme dans la géographie française au $X X^{e}$ siècle, Paris, L'Harmattan.

-, 2015, «Mai 68 et ses suites en géographie française», Revue d'histoire des sciences humaines, 26, p. 209-242. 
PAQUOT T., 2014, «Urbanisme, Urbanologie, études urbaines: I'improbable classification », Hermès, 67-3, p. 95-100.

PASSERON J.-C., 2006 (199I), Le Raisonnement sociologique: un espace non poppérien de l'argumentation, Paris, A. Michel.

PICHON M., LEININGER-FRÉZAL C. et DOUAY N., 2017, «La "professionnalisation" des formations en géographie: spécificité disciplinaire?», Carnets de géographes, 10, [en ligne]: <10.4000/cdg.| |64>.

POLLAK M., 1975, «L'efficacité par l'ambiguïté», Sociologie et Sociétés, 7-I, p. 29-49.

PUMAIN D., SANDERS L. et SAINT-JULIEN T., 1989, Villes et Auto-organisation, Paris, Economica.

ROBIC M.-C., 2003, «La ville, objet ou problème? La géographie urbaine en France (1890-1960)», Sociétés contemporaines, 49/50-I, p. 107.

-, 2007, «Recherche géographique: «Du génie perso au labo et au réseau », La Revue pour l'histoire du CNRS, 18, [en ligne, consulté le 10 janvier 2018]: $<$ http://journals.openedition.org/histoire-cnrs/395I>.

ROCHEFORT R., 1963, «Géographie sociale et sciences humaines », Bulletin de l'Association de géographes français, 314-315, p. 18-32.

VANT A., 1973, «Centre-ville et antagonismes sociaux: l'exemple stéphanois», Revue de géographie de Lyon, 48-I, p. 5-44.

-, 1981, Imagerie et urbanisation: recherches sur l'exemple stéphanois, Saint-Étienne, Centre d'études foréziennes.

-, 1984, «La géographie sociale lyonnaise en perspective », Géocarrefour, 59-3, p. $|3|-146$. 